\title{
CRITICAL LENGTHS FOR GLOBAL EXISTENCE OF SOLUTIONS FOR COUPLED SEMILINEAR SINGULAR PARABOLIC PROBLEMS*
}

\author{
BY \\ C. Y. CHAN ${ }^{1}$ (Lniversity of Southwestern Louisiana) \\ AND \\ C. S. CHEN (University of Nevada, Las Vegas)
}

1. Introduction. Let

$$
\begin{gathered}
\Omega=(0, a) \times\left(0, T_{a}\right), \\
\partial \Omega=([0, a] \times\{0\}) \cup\left(\{0, a\} \times\left(0, T_{a}\right)\right) .
\end{gathered}
$$

Here, $T_{a}(\leq \infty)$ denotes the largest value such that a solution $z<1$ exists for the following semilinear singular parabolic initial boundary value problem:

$$
L z=-(1-z)^{-1} \quad \text { in } \Omega, \quad z=0 \quad \text { on } \partial \Omega,
$$

where

$$
L \equiv \frac{\partial^{2}}{\partial x^{2}}+\frac{b}{x} \frac{\partial}{\partial x}-\frac{\partial}{\partial t}
$$

and $b$ is a constant less than 1 . If $T_{a}=\infty$, then $z(<1)$ exists globally. If $T_{a}<\infty$, then

$$
\lim _{t \rightarrow T_{a}^{-}} \max \{z(x, t): 0 \leq x \leq a\}=1^{-} .
$$

In this latter case, $z$ is said to quench at $T_{a}$ (cf. Acker and Walter [2]), and $T_{a}$ is called the quenching time. Recently, we [3] studied the critical length $a^{*}$ for the above problem such that $T_{a}=\infty$ for $a<a^{*}$, and $T_{a}<\infty$ for $a>a^{*}$; an upper bound for the quenching time is also determined. For further references, we refer to the above paper, and those written recently by Acker and Kawohl [1] and Chan and Kaper [4]. So far, only problems involving one scalar differential equation have been studied.

The main purpose here is to extend our study [3] on critical lengths for global existence of solutions and upper bounds for the quenching time for a scalar differential equation to the following coupled parabolic problem:

$$
\begin{gathered}
L u=-f(u) \text { in } \Omega, \\
u=0 \quad \text { on } \partial \Omega,
\end{gathered}
$$

${ }^{*}$ Received October 4, 1988.

${ }^{1}$ The work of this author was partially supported by the Board of Regents of the State of Louisiana under Grant LEQSF(86-89)-RD-A-11. 
where $u$ and $f(u)$ denote, respectively, the transpose of the vectors $\left(u_{1}, u_{2}\right)$ and $\left(f_{1}\left(u_{1}, u_{2}\right), f_{2}\left(u_{1}, u_{2}\right)\right)$. For brevity, we also use throughout this paper the convention that the subscript $i$ denotes the values 1 and 2, respectively. As in [3] and [4], we assume that the problem $(1.1)-(1.2)$ has a solution before its quenching time. In Sec. 2, we show that for global existence of the solution there exists a unique critical length $a^{*}$, which is determined by the steady state of the problem $(1.1)-(1.2)$ :

$$
\ell v=-f(v), \quad v(0)=0=v(a),
$$

where $v$ denotes the transpose of the vector $\left(v_{1}, v_{2}\right)$, and

$$
\ell \equiv \frac{d^{2}}{d x^{2}}+\frac{b}{x} \frac{d}{d x}
$$

In Sec. 3, we give a computational method for $a^{*}$ by using the Picard method and Green's function corresponding to the operator $-x^{b} /$. We construct a monotone increasing sequence converging componentwise uniformly to the minimal solution of the problem (1.3). By using differential inequalities, we determine an upper bound for $a^{*}$ to start the computational method. In Sec. 4, we use a comparison method to find a lower bound for the solution of the problem (1.1)-(1.2), and obtain an upper bound for the quenching time. Based on the theory developed in Sec. 3, we design, in Sec. 5, an algorithm to actually compute the critical length. For illustration of our computational method, an example is given in Sec. 6.

2. Existence of critical lengths. We impose the following conditions on $f$ :

(i) $f(0)>0$.

(ii) there exists a positive constant column vector $q$ with $q_{1} \geq q_{2}$ such that $f(w)$ is of class $C^{1}$ in $\left(-\infty, q_{1}\right) \times\left(-\infty, q_{2}\right)$, and

$$
\lim _{u_{2}^{\prime} \rightarrow q_{2}} f_{1}(w)=\infty
$$

(iii) to any $\epsilon$ with $0<\epsilon<q_{2}$, there exists a continuous function $C(x, t)$ which is bounded above such that

$$
f_{2}(w)-f_{1}(w) \geq C\left(w_{2}-w_{1}\right)
$$

for $w_{i} \leq q_{i}-\epsilon$.

(iv) the first partial derivatives of $f(w)$ are nonnegative and bounded for $w_{i} \leq$ $q_{i}-\epsilon$.

The quenching phenomenon is due to the singularity of $f_{1}$. The system quenches when $u_{2}$ reaches $q_{2}$ in a finite time.

First, let us investigate some properties of the solution of the problem (1.1)-(1.2).

LEMMA 1. (a) There exists at most one solution $u$ of the problem (1.1)-(1.2).

(b) The solution $u>0$ in $\Omega$.

(c) The solution $u$ is strictly increasing in $t$ for $0<x<a$.

Proof. (a) Let $w$ and $W$ be two distinct solutions. By the Mean Value Theorem,

$$
L\left(w_{i}-W_{i}\right)+\sum_{j=1}^{2} \frac{\partial f_{i}\left(\theta_{i}, \lambda_{i}\right)}{\partial W_{j}}\left(w_{j}-W_{j}\right)=0
$$


where $\left(\theta_{i}, \lambda_{i}\right)$ is a point on the line segment joining $w$ and $W$. By the maximum principle for weakly coupled parabolic systems (cf. Protter and Weinberger [5, p. 190]), $w-W=0$, and hence there exists at most one solution.

(b) By Condition (i) and (1.1),

$$
L u<-[f(u)-f(0)] .
$$

By the Mean Value Theorem,

$$
L u_{i}+\sum_{j=1}^{2} \frac{\partial f_{i}\left(\theta_{i}, \lambda_{i}\right)}{\partial u_{j}} u_{j}<0,
$$

where $\left(\theta_{i}, \lambda_{i}\right)$ is a point on the line segment joining $u$ and 0 . By the maximum principle for weakly coupled parabolic systems, $u>0$.

(c) Let

$$
u^{\delta}(x, t) \equiv u(x, t+\delta)-u(x, t),
$$

where $\delta>0$. From (1.1) and the Mean Value Theorem,

$$
L u_{i}^{\delta}+\sum_{j=1}^{2} \frac{\partial f_{i}\left(\theta_{i}, \lambda_{i}\right)}{\partial u_{j}} u_{j}^{\delta}=0
$$

where $\left(\theta_{i}, \lambda_{i}\right)$ is a point on the line segment joining $u(x, t+\delta)$ and $u(x, t)$. Since

$$
u^{\delta}(x, 0)=u(x, \delta)>0, \quad u^{\delta}(0, t)=0=u^{\delta}(a, t),
$$

it follows from the maximum principle for weakly coupled parabolic systems that $u^{\delta}>0$ in $\Omega$. Thus, $u$ is strictly increasing in $t$ for $0<x<a$.

LeMma 2. In $\Omega, u_{1} \leq u_{2}$.

Proof. By Condition (iii),

$$
L\left(u_{2}-u_{1}\right) \leq-C\left(u_{2}-u_{1}\right) .
$$

It follows from the strong maximum principle (cf. Protter and Weinberger [5, pp. 173-175]) that $u_{1} \leq u_{2}$ in $\Omega$.

We note that a solution of the problem (1.3) satisfies the following integral equation:

$$
v(x)=\int_{0}^{a} \xi^{b} G(x ; \xi) f(v(\xi)) d \xi
$$

where

$$
G(x ; \xi)= \begin{cases}\frac{x^{1-b}}{(1-b) a^{1-b}}\left(a^{1-b}-\xi^{1-b}\right), & 0 \leq x \leq \xi, \\ \frac{\xi^{1-b}}{(1-b) a^{1-b}}\left(a^{1-b}-x^{1-b}\right), & \xi \leq x \leq a,\end{cases}
$$

is Green's function associated with the operator $-x^{b} \ell$ subject to the first boundary conditions. 
THEOREM 3. If $u_{i} \leq q_{i}-\epsilon$, then the problem (1.3) has a solution $v$, to which $u$ converges componentwise uniformly from below as $t$ tends to infinity.

Proof. Let

$$
E(x, t) \equiv \int_{0}^{a} \xi^{b} G(x ; \xi) u(\xi, t) d \xi .
$$

From (2.1), $G(x ; \xi)>0$ for $x$ and $\xi$ in the interval $(0, a)$. Since $u<q$ in $\Omega$, it follows that for $b \neq-1$,

$$
\begin{aligned}
E(x, t) & <q \int_{0}^{a} \xi^{b} G(x ; \xi) d \xi \\
& =\frac{q}{(1-b) a^{1-b}}\left(\frac{1}{1+b}-\frac{1}{2}\right)\left(a^{2} x^{1-b}-a^{1-b} x^{2}\right) .
\end{aligned}
$$

Similarly for $b=-1$, we obtain

$$
E(x, t)<q \int_{0}^{a} \xi^{-1} G(x ; \xi) d \xi=\frac{q}{2} x^{2}(\ln a-\ln x) .
$$

We conclude that each component of $E(x, t)$ is bounded for $0 \leq x \leq a, 0<t$.

Since $u_{t} \geq 0$, we have

$$
E_{l}(x, t)=\int_{0}^{a} \xi^{b} G(x ; \xi) u_{t}(\xi, t) d \xi \geq 0 .
$$

It follows that

$$
E_{t}(x, t)=\int_{0}^{a} \xi^{b} G(x ; \xi)\left(u_{\xi \xi}+\frac{b}{\xi} u_{\xi}\right) d \xi+\int_{0}^{a} \xi^{b} G(x ; \xi) f(u(\xi, t)) d \xi .
$$

Let us consider the first integral on the right-hand side of (2.2). Using integration by parts, we have

$$
\begin{aligned}
\int_{0}^{a} \xi^{h} G(x ; \xi)\left(u_{\xi \xi}+\frac{b}{\xi} u_{\xi}\right) d \xi \\
=\int_{0}^{a} G(x ; \xi)\left(\xi^{b} u_{\xi}\right)_{\xi} d \xi \\
=\frac{1}{(1-b) a^{1-h}}\left[\left(a^{1-h}-x^{1-b}\right) x u_{x}(x, t)-(1-b)\left(a^{1-b}-x^{1-h}\right) u(x, t)\right. \\
\left.=-u(x, t) . \quad-x\left(a^{1-b}-x^{1-h}\right) u_{x}(x, t)-x^{1-b}(1-b) u(x, t)\right]
\end{aligned}
$$

From (2.2), we have

$$
E_{t}(x, t)=-u(x, t)+\int_{0}^{a} \xi^{h} G(x ; \xi) f(u(\xi, t)) d \xi .
$$

Because each component of the integrand in (2.3) is monotone increasing with respect to $t$, it follows from the Monotone Convergence Theorem (cf. Royden [6, p. 84]) that

$$
J(x)=-v(x)+\int_{0}^{a} \xi^{h} G(x ; \xi) f(v(\xi)) d \xi,
$$


where $J(x) \equiv \lim _{t \rightarrow \infty} E_{t}(x, t)$, and $v(x)=\lim _{t \rightarrow \infty} u(x, t)$. Since $E_{t}(x, t) \geq 0$, we have $J(x) \geq 0$. Let us assume that for some $x$, one of the components of $J(x)$ is positive. It follows that its corresponding component of $E(x, t)$ would increase without bound as $t$ tends to infinity. This contradicts the fact that each component of $E(x, t)$ is bounded. Thus, $J(x)=0$. From (2.4),

$$
v(x)=\int_{0}^{a} \xi^{b} G(x ; \xi) f(v(\xi)) d \xi .
$$

A direct computation shows that $v$ is twice differentiable at any given $x$ in $(0, a)$, and forms a solution of the problem (1.3). The uniform convergence follows from Dini's Theorem.

The following theorem compares solutions corresponding to different values of $a$.

Theorem 4. Let $u(x, t ; a)$ denote a solution of the problem (1.1)-(1.2). For any positive number $\eta, u(x, t ; a)<u(x, t ; a+\eta)$ in $\Omega$; furthermore,

$$
u_{i}(x, t ; a)<\min \left\{u_{i}(x+\sigma, t ; a+\eta): 0 \leq \sigma \leq \eta\right\} \quad \text { in } \Omega .
$$

Proof. Let

$$
u^{\eta}(x, t) \equiv u(x+\sigma, t ; a+\eta)-u(x, t ; a) .
$$

By the Mean Value Theorem,

$$
L u_{i}^{\eta}+\sum_{j=1}^{2} \frac{\partial f_{i}\left(\theta_{i}, \lambda_{i}\right)}{\partial u_{j}} u_{j}^{\eta}=0,
$$

where $\left(\theta_{i}, \lambda_{i}\right)$ is a point on the line segment joining $u(x+\sigma, t ; a+\eta)$ and $u(x, t ; a)$. Since

$$
u^{\eta}(x, 0)=0, \quad u^{\eta}(0, t)=u(\sigma, t ; a+\eta) \geq 0, \quad u^{\eta}(a, t)=u(a+\sigma, t ; a+\eta) \geq 0,
$$

the Theorem then follows by using the maximum principle.

From Theorems 3 and 4, the critical length $a^{*}$ for global existence is determined by the supremum of all values $a(>0)$ such that a solution $v$ of the problem (1.3) exists. From Lemma 2 and Theorem 3, we have $v_{1}(x) \leq v_{2}(x)$ for $0 \leq x \leq a$. It suffices to find the supremum of all values $a$ such that $v_{2}<q_{2}$.

3. Computational method of critical lengths. In this section, we employ the monotone iterative method to construct a sequence converging componentwise to the minimal steady-state solution. We use the Picard method to construct this monotone sequence $\left\{v^{n}: n=0,1,2, \ldots\right\}: v^{0}=0$, and for $n=0,1,2, \ldots$,

$$
\begin{gathered}
\ell v^{n+1}=-f\left(v^{n}\right), \\
v^{n+1}(0)=0=v^{n+1}(a) .
\end{gathered}
$$

Thus,

$$
v^{n+1}(x)=\int_{0}^{a} \xi^{h} G(x ; \xi) f\left(v^{n}(\xi)\right) d \xi .
$$

Our next result (cf. Theorem 5 of Chan and Chen [3]) gives existence of the steady-state solution constructively. 
THEOREM 5. For $a<a^{*}$, the nonlinear singular two-point boundary value problem (1.3) has the minimal solution $\mathbf{v}$, to which the sequence $\left\{v^{n}\right\}$ converges componentwise monotonically upwards for $0<x<a$; furthermore, $u(x, t) \leq \mathbf{v}(x)$ in $\Omega$ with $T_{a}=\infty$.

Proof. From (3.1),

$$
\ell v^{1}=-f(0)<0 .
$$

It follows from (3.3) that $v^{1}>0$ for $0<x<a$. From Lemma 1 (b) and Theorem 3, $v>0$ for $0<x<a$. Since

$$
\ell\left(v-v^{1}\right)=-[f(v)-f(0)] \leq 0,
$$

we have $v^{1} \leq v$. Let us assume that for some value of $n$, say $j(\geq 1)$,

$$
0 \leq v^{j-1} \leq v^{j} \leq v, \quad 0<x<a .
$$

Then

$$
\begin{gathered}
\ell\left(v^{j+1}-v^{j}\right)=-\left[f\left(v^{j}\right)-f\left(v^{j-1}\right)\right] \leq 0 \\
\ell\left(v-v^{j+1}\right)=-\left[f(v)-f\left(v^{j}\right)\right] \leq 0 .
\end{gathered}
$$

It follows that

$$
0 \leq v^{j-1} \leq v^{j} \leq v^{j+1} \leq v, \quad 0<x<a .
$$

By the principle of mathematical induction, $\left\{v^{n}\right\}$ converges componentwise monotonically upwards. Since the sequence is bounded above and below, there exists a function $\mathbf{v}(x)$ such that $\lim _{n \rightarrow \infty} v^{n}=\mathbf{v}$. Because each component of the integrand in (3.3) is nonnegative and increasing, it follows from the Monotone Convergence Theorem that

$$
\mathbf{v}(x)=\int_{0}^{a} \xi^{b} G(x ; \xi) f(\mathbf{v}(\xi)) d \xi .
$$

This implies that $\mathbf{v}$ is the solution of (1.3). Because of (3.4), $\mathbf{v}$ is the minimal solution.

By the Mean Value Theorem,

$$
L\left(\mathbf{v}_{i}-u_{i}\right)+\sum_{j=1}^{2} \frac{\partial f_{i}\left(\theta_{i}, \lambda_{i}\right)}{\partial u_{j}}\left(\mathbf{v}_{j}-u_{j}\right)=0 \quad \text { in } \Omega,
$$

where $\left(\theta_{i}, \lambda_{i}\right)$ is a point on the line segment joining $\mathbf{v}$ and $u$. Since $\mathbf{v}-u \geq 0$ on $\partial \Omega$, it follows from the maximum principle for weakly coupled parabolic systems that $u(x, t) \leq \mathbf{v}(x)$ in $\Omega$ with $T_{a}=\infty$.

For each $n \geq 1$, the components of $v^{n}(x)$ may have different critical points, and these points may also vary as $n$ changes. The next result shows that each component $v_{i}^{n}(x)$ has a unique maximum in the interval $(0, a)$. This property enables one to use the subroutine DUVMGS from the IMSL Library (Version 1.0, April, 1987; MALB-USM-PERFCT-1.0) to find, to double precision, the maximum of $v_{2}^{n}(x)$ easily (without having to give an initial guess where its critical point is). 
LFMMA 6. For $a<a^{*}$ and each $n \geq 1$, the function $v_{i}^{n}(x)$ has a unique (positive) maximum.

Proof. Since $v^{n}(x)$ is nonnegative for each $n \geq 1$, it follows from (3.2) that $v_{i}^{n}(x)$ attains its maximum somewhere in the interval $(0, a)$. If $h_{i}$ is its critical point, then it follows from (3.1) and Conditions (i) and (iv) that

$$
v_{i}^{n^{\prime \prime}}\left(h_{i}\right)=-f_{i}\left(v^{n-1}\left(h_{i}\right)\right)<0,
$$

which shows that all critical points of $v_{i}^{n}(x)$ give relative maxima. Hence, there can only be one maximum, and the Lemma is proved.

From this lemma, if $\max _{0 \leq x \leq a} v_{2}^{n}(x)>q_{2}$ for some $n$, then the parabolic system does not have a global solution; otherwise, its solution exists for all $t>0$. For computation, we need the following condition:

$(\mathbf{F})$ for $w_{i} \leq q_{i}-\varepsilon$, either $\partial f_{2}(w) / \partial w_{2}>0$, or both $\partial f_{1}(x) / \partial w_{2}$ and $\partial f_{2}(w) / \partial w_{1}$ are positive.

Following the proof of Theorem 5 , we obtain for $v_{i} \leq q_{i}-\varepsilon$, and $j=1,2,3, \ldots$,

$$
0<v_{2}^{j}<v_{2}^{j+1}<v_{2} \text { for } 0<x<a \text { if } \partial f_{2}(v) / \partial v_{2}>0
$$

$0<v^{j}<v^{j+1}<v$ for $0<x<a$ if $\partial f_{1}(v) / \partial v_{2}$ and $\partial f_{2}(v) / \partial v_{1}$ are positive.

By using these inequalities, a proof similar to that of Lemma 6 gives the following result.

LEMMA 7. For $a<a^{*}$ and each $n \geq 0$, if Condition (F) holds, then the difference $v_{2}^{n+1}(x)-v_{2}^{n}(x)$ between two successive iterates constructed by (3.1)-(3.2) has a unique (positive) maximum.

Lemma 7 may be used to stop the computation of the minimal solution $\mathbf{v}$ if the maximum of the difference $v_{2}^{n+1}(x)-v_{2}^{n}(x)$ between two successive iterates is within the tolerance error.

Obviously, 0 is a lower bound for the critical length. To obtain an upper bound $a_{u}$ for the critical length, let us consider the following scalar problem:

$$
V^{\prime \prime}+\frac{b}{x} V^{\prime}=-f_{2}(0), \quad V(0)=0=V(a) .
$$

It follows from the strong maximum principle that $V<v_{2}$.

For $b \neq-1$,

$$
V(x)=\frac{f_{2}(0) x^{2}}{2(1+b)}\left[\left(\frac{a}{x}\right)^{1+b}-1\right] .
$$

Differentiating $V(x)$ with respect to $x$ yields

$$
V^{\prime}(x)=\frac{f_{2}(0)}{(1+b)}\left(\frac{1-b}{2} a^{1+b} x^{-b}-x\right)
$$

from which $V^{\prime}(x)=0$ occurs at $x=a[(1-b) / 2]^{(1+b)^{-1}}$, where $V^{\prime \prime}<0$. Thus, the absolute maximum of $V(x)$ occurs at $x=a[(1-b) / 2]^{(1+b)^{-1}}$. An upper bound for the critical length $a_{u}$ such that $V$ attains $q_{2}$ at this point is given by

$$
q_{2}=f_{2}(0) 2^{-(3+b) /(1+b)}(1-b)^{(1-b) /(1+b)} a_{u}^{2} .
$$


Thus,

$$
a_{u}=\left[\frac{q_{2}}{f_{2}(0)} 2^{(3+b) /(1+b)}(1-b)^{(b-1) /(1+b)}\right]^{1 / 2} \text { for } b \neq-1 .
$$

For $b=-1$, the solution of the problem (3.5) is given by

$$
V(x)=\frac{f_{2}(0)}{2} x^{2}(\ln a-\ln x) .
$$

Thus,

$$
V^{\prime}(x)=f_{2}(0) x\left(\ln a-\ln x-\frac{1}{2}\right),
$$

from which $V^{\prime}(x)=0$ gives $x=a e^{-1 / 2}$, where $V^{\prime \prime}<0$. It follows that $V(x)$ attains its maximum at $x=a e^{-1 / 2}$. Therefore, an upper bound $a_{u}$ such that $V$ attains $q_{2}$ at this point is given by

$$
q_{2}=\frac{f_{2}(0) a_{u}^{2}}{4 e}
$$

Thus,

$$
a_{u}=2\left[\frac{e q_{2}}{f_{2}(0)}\right]^{1 / 2} \quad \text { for } b=-1 .
$$

We have the following result.

LEMMA $8.0<a^{*} \leq a_{u}$, where $a_{u}$ is given by (3.6) or (3.7).

4. Quenching time. From Lemma $2, u_{1} \leq u_{2}$ in $\Omega$. It follows from Condition (iv) that

$$
L u_{1}=-f_{1}(u) \leq-\Psi\left(u_{1}\right)
$$

where $\Psi\left(u_{1}\right)=f_{1}\left(u_{1}, u_{1}\right)$. By the strong maximum principle, the solution $U$ of the scalar problem,

$$
L U=-\Psi(U) \text { in } \Omega, \quad U=0 \text { on } \partial \Omega,
$$

is a lower bound of $u_{1}$. On the other hand, a lower bound $\omega$ of $U$ by the strong maximum principle is given by the problem:

$$
\begin{gathered}
L \omega \geq-\Psi(\omega) \text { in } \Omega, \\
\omega=0 \quad \text { on } \partial \Omega .
\end{gathered}
$$

If $\omega$ quenches, then certainly $u$ quenches.

Let us seek a lower bound $\omega$ in the form: $\rho(x) \mu(t)$, where $\rho(x)=x^{\prime \prime} J_{\nu^{\prime}}\left(j_{\nu}, x / a\right)$ with $\nu=(1-b) / 2$, and $j_{\nu}$, denoting the first positive zero of the Bessel function $J_{1}$ of the first kind of order $\nu$. We note that $\rho$ is the solution of the scalar problem:

$$
\ell \rho=-\left(j_{\nu} / a\right)^{2} \rho, \quad \rho(0)=0=\rho(a) .
$$

From (4.1), we have the following inequality:

$$
\rho^{\prime \prime} \mu+\frac{b}{x} \rho^{\prime} \mu-\rho \mu^{\prime} \geq-\Psi(\rho \mu) .
$$

It follows that

$$
\mu^{\prime}+\left(\frac{j_{\nu}}{a}\right)^{2} \mu \leq \frac{\Psi(\rho \mu)}{\rho}
$$


Since the left-hand side of the inequality does not depend on $x$, we can obtain a lower bound for $\mu$ by taking the infimum of the right-hand side with respect to $x$. Let us denote this infimum by $p(\mu(t))$. We may then determine $\mu(t)$ by considering the initial value problem:

$$
\mu^{\prime}(t)+\left(\frac{j_{\nu}}{a}\right)^{2} \mu(t)=p(\mu(t)), \quad \mu(0)=0,
$$

where the initial condition comes from $u_{1}(x, 0)=0$. Now, $\rho$ attains its maximum at $x=a j_{\nu-1} / j_{\nu}$. Let $m$ denote this maximum value. Then, the time $t_{1}$ at which $m \mu\left(t_{1}\right)=q_{2}$ is an upper bound for the quenching time $T_{a}$.

5. Numerical algorithm. We apply the results in Sec. 3 to compute critical lengths for global existence of solutions for various given $b$ and $f$ satisfying Condition (F). By Lemma 8 , an upper bound $a_{u}$ of the critical length $a^{*}$ can be determined from the data: $b, f_{2}(0)$, and $q_{2}$. We can take 0 and $a_{u}$ as the starting points for finding $a^{*}$. By the method of bisection, we approximate $a^{*}$ by $a^{* *}=a_{u} / 2$, and evaluate $v^{n+1}(x)$ by using the representation formula (3.3) with $v^{0}=0$. By Lemma 6 , we can use the subroutine DUVMGS to find $\max _{0 \leq x \leq a} v_{2}^{n+1}(x)$. If this maximum is greater than or equal to $q_{2}$ for some $n$, then we replace $a_{u}$ by $a^{* *}$; otherwise, $u$ exists globally, and we replace 0 by $a^{* *}$. We repeat the above procedure of bisection until the demanded accuracy is reached; for example, the difference between two successive approximations of $a^{*}$ is less than $5 \times 10^{-(r+1)}$. Since the difference between $a^{*}$ and its approximation $a^{* *}$ can be made as small as we want, $a^{* *}$ can be taken numerically to be $a^{*}$.

In order to compute $v^{n+1}(x)$, we adopt four subroutines from the IMSL Library: DQDAG (to integrate, to double precision, a function by using a globally adaptive scheme based on Gauss-Kronrod rules), DCSINT (to compute, to double precision, the cubic spline interpolant with the 'not-a-knot' condition), DCSVAL (to evaluate, to double precision, a cubic spline at a given point), and DUVMGS. We use the following steps:

1. We subdivide the interval $0 \leq x \leq a^{* *}$ into 20 equal subintervals: $0=x_{1}<$ $x_{2}<x_{3}<\cdots<x_{21}=a^{* *}$.

2. At the nineteen interior subdivision points, we evaluate

$$
\begin{gathered}
y_{1}(x)=(1-b)^{-1}(x / a)^{1-b}, \\
y_{2}(x)=(1-b)^{-1}\left[1-(x / a)^{1-b}\right],
\end{gathered}
$$

and store them in the memory of the computer for future use.

3. Let

$$
\begin{gathered}
F_{1 n}\left(x_{j}, x_{k}\right) \equiv \int_{x_{j}}^{x_{k}} \xi f\left(v^{n}(\xi)\right) d \xi, \\
F_{2 n}\left(x_{j}, x_{k}\right) \equiv \int_{x_{j}}^{x_{k}}\left(\xi^{b} a^{1-b}-\xi\right) f\left(v^{n}(\xi)\right) d \xi .
\end{gathered}
$$

To save computer time in obtaining $v^{n+1}$ from $v^{n}$, we evaluate $v^{n+1}\left(x_{11}\right)$ first. From (3.3),

$$
v^{n+1}\left(x_{11}\right)=y_{2}\left(x_{11}\right) F_{1 n}\left(x_{1}, x_{11}\right)+y_{1}\left(x_{11}\right) F_{2 n}\left(x_{11}, x_{21}\right) .
$$


To obtain $v^{n+1}\left(x_{10}\right)$, we only need to compute $F_{1 n}\left(x_{10}, x_{11}\right)$ and $F_{2 n}\left(x_{10}, x_{11}\right)$ since $v^{n+1}\left(x_{10}\right)=y_{2}\left(x_{10}\right)\left[F_{1 n}\left(x_{1}, x_{11}\right)-F_{1 n}\left(x_{10}, x_{11}\right)\right]+y_{1}\left(x_{10}\right)\left[F_{2 n}\left(x_{11}, x_{21}\right)+F_{2 n}\left(x_{10}, x_{11}\right)\right]$. We can thus successively compute $v^{n+1}$ at $x_{10}, x_{9}, x_{8}, \ldots, x_{2}$. Similarly, $v^{n+1}\left(x_{12}\right)=y_{2}\left(x_{12}\right)\left[F_{1 n}\left(x_{1}, x_{11}\right)+F_{1 n}\left(x_{11}, x_{12}\right)\right]+y_{1}\left(x_{12}\right)\left[F_{2 n}\left(x_{11}, x_{21}\right)-F_{2 n}\left(x_{11}, x_{12}\right)\right]$. Proceeding in this way, we obtain successively $v^{n+1}$ at $x_{12}, x_{13}, x_{14}, \ldots, x_{20}$.

4. We stop the computation of $v^{n+1}$ in two ways:

(a) if $\max _{0 \leq x \leq a} v_{2}^{n}(x) \geq q_{2}$, then $a>a^{*}$.

(b) if $\max _{0 \leq x \leq a} v_{2}^{n}(x)<q_{2}$, and $\max _{0 \leq x \leq a}\left[v_{2}^{n+1}(x)-v_{2}^{n}(x)\right]<5 \times 10^{-(r+1)}$, then $u$ exists globally.

6. Example. Let us consider the following coupled semilinear singular parabolic problem:

$$
L u=\left\{\begin{array}{l}
-\frac{1}{1-u_{2}}-k_{1} u_{1} \\
-\frac{1}{1-u_{1}}-k_{2} u_{2}
\end{array} \quad \text { in } \Omega, \quad u=0 \quad \text { on } \partial \Omega .\right.
$$

Here, $0 \leq k_{1}<k_{2}$. (If $k_{1}=k_{2}$, then it follows from the strong maximum principle that $u_{1} \equiv u_{2}$.) We note that Conditions (i)-(iv) and (F) are satisfied. From Lemma 2 , we have $u_{1} \leq u_{2}$. To estimate the quenching time, we use

$$
\Psi\left(u_{1}\right)=\frac{1}{1-u_{1}}+k_{1} u_{1} .
$$

In this case,

$$
\frac{\Psi(\rho \mu)}{\rho}=\frac{1}{\rho(1-\rho \mu)}+k_{1} \mu
$$

Hence,

$$
\mu^{\prime}+\left(\left(\frac{j_{\nu}}{a}\right)^{2}-k_{1}\right) \mu \leq \frac{1}{\rho(1-\rho \mu)},
$$

which is (2.5) of Chan and Chen [3] with $\left(j_{\nu} / a\right)^{2}$ replaced by $\left(j_{\nu} / a\right)^{2}-k_{1}$. It follows from the discussion there that an upper bound $t_{1}$ of the quenching time is given by

$$
t_{1}=\left(\frac{1}{\beta(4-\beta)}\right)^{1 / 2} \tan ^{-1}\left(\frac{\beta}{4-\beta}\right)^{1 / 2}-\frac{1}{2 \beta} \ln \left(1-\frac{\beta}{4}\right)+\frac{\ln 2}{4-\beta} \text {. }
$$

where

$$
\beta=\left(\frac{j_{\nu 1}}{a}\right)^{2}-k_{1}
$$

Results (rounded to 4 decimal points) for various $b, k_{1}$, and $k_{2}$ (with $r=5$ ) are given in the following table. We note that when $k_{1}$ and $k_{2}$ are both zero, we reproduce the results of Table 1 of Chan and Chen [3]; in particular, when $b, k_{1}$, and $k_{2}$ are all zero, we reproduce the results $a^{*}=1.5303$ of Walter [7]. 
TABLE 1. Critical lengths corresponding to different values of $b, k_{1}$, and $k_{2}$, and upper bounds for quenching time when $a=\pi$.

\begin{tabular}{|c|c|c|c|c|c|}
\hline$b$ & $\nu$ & $k_{1}$ & $k_{2}$ & $a^{*}$ & $t_{1}$ \\
\hline & & & 0.75 & 1.5445 & \\
\hline \multirow{3}{*}{-0.4} & 0.7 & 0.5 & 1.00 & 1.5238 & 0.6668 \\
\hline & & & 1.25 & 1.5031 & \\
\hline & & & 0.75 & 1.4274 & \\
\hline \multirow[t]{3}{*}{0} & 0.5 & 0.5 & 1.00 & 1.4081 & 0.6385 \\
\hline & & & 1.25 & 1.3887 & \\
\hline & & & 0.75 & 1.2994 & \\
\hline \multirow[t]{3}{*}{0.4} & 0.3 & 0.5 & 1.00 & 1.2818 & 0.6145 \\
\hline & & & 1.25 & 1.2641 & \\
\hline & & & 0.00 & 1.6544 & \\
\hline \multirow[t]{3}{*}{-0.4} & 0.7 & 0 & 0.50 & 1.6058 & 0.7099 \\
\hline & & & 1.00 & 1.5576 & \\
\hline & & & 0.00 & 1.5303 & \\
\hline \multirow[t]{3}{*}{0} & 0.5 & 0 & 0.50 & 1.4848 & 0.6772 \\
\hline & & & 1.00 & 1.4397 & \\
\hline & & & 0.00 & 1.3935 & \\
\hline \multirow[t]{2}{*}{0.4} & 0.3 & 0 & 0.50 & 1.3519 & 0.6496 \\
\hline & & & 1.00 & 1.3106 & \\
\hline
\end{tabular}

Acknowledgment. The authors thank the referee for the helpful comments.

\section{REFERENCES}

[1] A. Acker and B. Kawohl, Remarks on quenching, Nonlinear Anal. 13, 53-61 (1989)

[2] A. Acker and W. Walter, On the global existence of solutions of parabolic differential equations with a singular nonlinear term, Nonlinear Anal. 2, 499-505 (1978)

[3] C. Y. Chan and C. S. Chen, A numerical method for semilinear singular parabolic quenching problems, Quart. Appl. Math. 47, 45-57 (1989)

[4] C. Y. Chan and H. G. Kaper, Quenching for semilinear singular parabolic problems, SIAM J. Math. Anal. 20, 558-566 (1989).

[5] M. H. Protter and H. F. Weinberger, Maximum Principles in Differential Equations, Prentice Hall, Englewood Cliffs, 1967, pp. 173-175, and 190

[6] H. L. Royden, Real Analysis, 2nd ed., Macmillan Publishing Co., New York, 1968, p. 84

[7] W. Walter, Parabolic differential equations with a singular nonlinear term, Funkcial. Ekvac. 19, 271277 (1976) 